\title{
Interference with memory for lifted weight'
}

\author{
ALLEN PARDUCCI, LOUISE M. MARSHALL AND MARY DEGNER \\ UNIVERSITY OF CALIFORNIA, LOS ANGELES
}

An adaptation-level model for memory was tested by interpolating different weights between the standard and comparison weights. The results suggest that the model should be modified to account for the general tendency to use alternative categories of judgment with equal frequency. In terms of the modified model, memory for the standard shifts toward the value of the interfering stimulus, the magnitude of shift being proportional to the difference between the interfering stimulus and the value the memory would otherwise have had.

How is memory for a simple psychophysical stimulus affected by similar, interfering stimuli? One answer is suggested by the theory of adaptation level (Helson, 1964). It seems within the spirit of Helson's approach to identify the remembered value of a stimulus with its adaptation level. Since the theory asserts that adaptation level is a weighted mean of all stimuli in the psychological frame of reference, it implies that memory shifts toward the value of each of the stimuli that affect it.

The identification of memory with adaptation level is itself suggested by psychophysical research using the method of constant stimulus differences. The typical finding has been that the value of the comparison stimulus judged equal to a previously presented standard varies directly with the values of other stimuli in the same experimental situation (Guilford, 1954, pp. 310311). The present research provides a basis for further development of this interpretation of adaptation-level theory.

\section{METHOD}

Weights were presented by the method of constant stimulus differences, with an unjudged weight interpolated between each presentation of the standard and comparison weights. The standard was always $109 \mathrm{gm}$, and the same six comparison weights, 84, 93, 101, 109,117 , and $125 \mathrm{gm}$., were used in all experimental conditions. The weights were lifted by pulling down a handle. A cord led from the handle to the weight, passing through a partition which blocked S's view of both $E$ and the weight. The instructions stated that $S$ was to lift three weights on each trial, "...judging the third as either heavier or lighter than the first, the standard. You have to lift the second weight, but you are not to judge it."

Seven groups, of 10 Ss each, were presented the different combinations of interpolated weights shown in Table 1. Four of the groups were presented two different interpolated weights, each weight being interpolated on half the trials. Thus, for the 93-190 condition, the trials began with the 109-gm. standard, which was followed on half the trials by the 93-gm. interpolation and then one of the six comparison weights; the 190-gm. interpolation was used for the remaining trials. The sequence of trials was random, with the restriction that each interpolated weight preceded each of the six comparison weights four times in each block of 48 trials. For the 93-Only and 190Only conditions, the same interpolated weight was used on every trial. There was no interpolated weight for the Control condition. The temporal intervals were as brief as possible, limited only by S's preferred rate of lifting and E's dexterity at changing weights. To keep the same inter-stimulus intervals for the Control condition, E attached an unlifted, interpolated weight on every trial. Each $S$ served for approximately two hours, including a short rest between each of the seven, 48-trial blocks.

\section{RESULTS AND DISCUSSION}

\section{Consistent data}

Figure 1 illustrates the treatment of the results, using the data from the 93-190 condition. The proportion of "heavier" judgments for each of the six comparison weights is shown separately for the $93-\mathrm{gm}$. and the $190-\mathrm{gm}$. interpolations. When these 12 proportions are plotted as normal deviates, the cumulative-normal psychometric functions are best fitted by straight lines. Although a logarithmic transformation of the stimulus values would better reflect their psychological differences, the changes in their relative differences would be so small that none of the conclusions would be affected significantly. The traditional point of subjective equality (PSE) is shown for each interpolation, PSE being that comparison value that would be judged "heavier" for $50 \%$ of its presentations.

Table 1. Mean and Standard Error of the PSEs and SDs for each Interpolated Weight (gm.)

\begin{tabular}{|c|c|c|c|c|c|c|}
\hline \multirow[t]{2}{*}{ Condition } & \multirow[t]{2}{*}{ Interp. Weight } & \multicolumn{2}{|c|}{ PSE } & \multicolumn{2}{|c|}{ SD } & \multirow[t]{2}{*}{$M_{1}{ }^{\alpha}$} \\
\hline & & Mean & SE & Mean & SE & \\
\hline \multirow[t]{2}{*}{$93-190$} & 93 & 96.9 & .89 & 15.7 & 1.17 & 96.2 \\
\hline & 190 & 114.3 & 2.64 & 16.1 & .74 & 113.9 \\
\hline \multirow[t]{2}{*}{$109 \cdot 162$} & 109 & 97.8 & 1.10 & 15.4 & .67 & 98.8 \\
\hline & 162 & 107.4 & 1.75 & 17.9 & 1.47 & 108.5 \\
\hline \multirow[t]{2}{*}{$125-141$} & 125 & 101.1 & 1.47 & 15.9 & 1.42 & 101.6 \\
\hline & 141 & 104.8 & 1.77 & 15.8 & 1.65 & 104.6 \\
\hline \multirow[t]{2}{*}{$77-93$} & 77 & 99.6 & .89 & 14.6 & .80 & 90.4 \\
\hline & 93 & 104.6 & .95 & 13.7 & .97 & 93.4 \\
\hline Control & 0 & 103.8 & .83 & 13.0 & .62 & 94.0 \\
\hline 93-Only & 93 & 103.4 & 1.60 & 14.3 & 1.08 & 93.8 \\
\hline 190-Only & 190 & 110.8 & 2.31 & 16.2 & .97 & 116.4 \\
\hline
\end{tabular}

$a_{\text {Remembered value of the standard as fitted by the frequency }}$ model. 


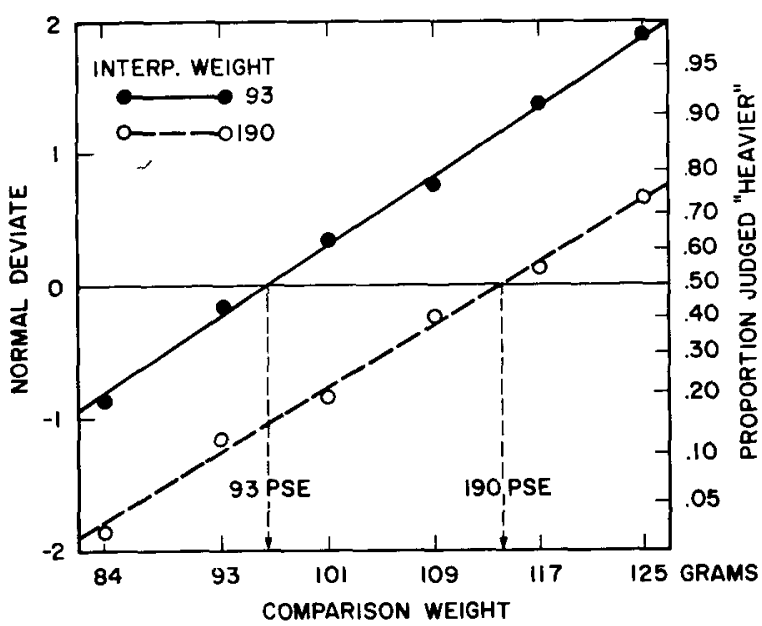

Fig. 1. Normal deviates corresponding to the proportions of "heavier" judgments of the comparison weights in the 93-190 condition, tabulated separately for trials using each of the two interpolated weights.

If memory for the standard is identified with adaptation level, the remembered value is simply the PSE which Helson uses as his measure of adaptation level. From Helson's presentation (1964, pp. 125-231), one would describe the effects of interpolated values with a simple averaging model. In this model, the PSE for a trial is a weighted mean of the weight interpolated on that trial $\left(I_{1}\right)$, the other interpolated weight $\left(I_{2}\right)$, and some value (P) representing the pooled effect of the standard, comparison stimuli, and any other stimuli common to all of the experimental conditions:

$$
\mathrm{PSE}_{1}=a \mathrm{I}_{1}+b \mathrm{I}_{2}+c \mathrm{P} \text {, }
$$

where $a+b+c=1$. Since the values of $I_{1}$ and $I_{2}$ are reversed for trials on which $\mathrm{I}_{2}$ is the interpolated weight, the averaging model predicts that the difference between the two PSEs for each condition is directly proportional to the difference between the two interpolated weights.

Figure 1 shows that PSE tabulated for the grouped data is markedly lower when the interpolated weight is $93 \mathrm{gm}$. than when it is $190 \mathrm{gm}$. The means of the PSEs for each interpolated value, computed separately by least squares for each $S$, are shown in Table 1 . The difference between the two PSEs for each of the four conditions using two interpolated weights is in the expected direction. The magnitudes of these differences are proportional to the differences between the interpolated weights and thus consistent with the adaptationlevel interpretation of memory.

The slopes of the psychometric functions in Fig. 1 are approximately equal, as are the slopes for all of the experimental conditions. This measure of sensitivity is presented in Table 1 as the SD, the reciprocal of slope. The similarity of the SDs provides a second basis for evaluating the simple averaging model as an interpretation of memory. For assuming that the normal deviate corresponding to the proportion of "heavier" judgments is directly proportional to the difference between the comparison stimulus and PSE, the slope is the constant of proportionality. The model's prediction that the slope remains constant can be tested by analysis of variance, the prediction being that there is no interaction between the effects of the interpolated and the comparison stimuli. Separate analyses were performed for each of the first four groups in Table 1, using the 12 normal deviates from each $\mathrm{S}$ as raw scores. Although the main effect of the interpolated stimulus was significant in each analysis $(p<.01)$, none of the four interactions approached statistical significance $(p>.05)$.

The constancy of SD recalls the constancy of d', the measure of sensitivity used in the theory of signal detectability (Swets, Tanner, \& Birdsall, 1961). Like SD, $d '$ appears to be largely independent of various features of the context that change the criterion for threshold judgments. A similar constancy is found with absolute category judgments, such as "heavy" and "light" for which there is no standard (Parducci, 1965). Changes in the stimulus frequencies have dramatic effects upon the location of the category limens, but SDs are scarcely affected.

Separate tabulation was made of the judgments of each comparison stimulus as a function of the comparison stimulus that immediately precededit. As demonstrated long ago using the present method but no interpolations (Fernberger, 1920), judgments vary inversely with the value of the preceding comparison stimulus. This small but systematic sequential effect is consistent with an averaging model, requiring only that the more recently presented values be weighted more heavily. However, the effective dependency extends only to the immediately preceding comparison stimulus, judgments appearing to be independent of which of the two interpolated values has been presented on the preceding trial.

\section{Inconsistent data}

Although the data from the first four groups in Table 1 are consistent with the adaptation-level interpretation of memory, the direction of some of the differences between the PSEs for these conditions and those using a single interpolated weight are not. Insofar as prior 190-gm. interpolations affect the PSE on the 93-gm. trials for the 93-190 condition, this PSE should be higher than the PSE for the 93-Only condition. The difference is statistically significant $(p<.01)$ but in the opposite direction. For the same reason, the finding that PSE for the 190-Only condition is lower than PSE for the $190-\mathrm{gm}$. trials in the $\mathbf{9 3 - 1 9 0}$ condition is also contrary to adaptation-level theory.

Does the interpolated weight interfere less with memory when it is always the same value than when it varies from trial to trial? The PSE for 93-Only is virtually identical to the PSE for the Control condition, in which there was no interpolation, both showing the usual negative time-order error. In terms of the theory 
of adaptation level, the contradictions described in the preceding paragraph could be handled by a difference in weighting that reflected a possible difference in attention: the interpolation might be given less weighting when it does not vary from trial to trial. However, this would not account for the finding that the PSE following a $93-\mathrm{gm}$. interpolation is significantly higher for the 77-93 than for the 93-190 condition $(p<.001)$. The interpolations for these two conditions should have the same weighting since each has two different interpolated values. The adaptation-level model requires that the PSE following a 93-gm. interpolation be lowered by any residual effect of the $77-\mathrm{gm}$. interpolation and that it be raised by any residual effect of the $190-\mathrm{gm}$. interpolation. Since the difference between these two PSEs is in the opposite direction, it is inconsistent with both the simple weighted-average interpretation of the theory of adaptation level and also with the modified interpretation which assumes that the single interpolated stimulus has less affect upon memory.

These contradictions appear to be examples of the general failure of the adaptation-level approach to account for response effects. The tendency for $S$ to use the categories of judgment with equal frequency seems particularly apparent with absolute judgments (Parducci, 1965). However, it is also suggested by experiments with comparative judgments (Harris, 1948).

Consider how this tendency toward equal frequency applies to the present data. As shown in Fig. 1, there was a preponderance of "heavier" judgments on trials with the 93-gm. interpolation. This was balanced by the preponderance of "lighter" judgments on trials with the $190-\mathrm{gm}$. interpolation. But the preponderance of "heavier" judgments was instead increased when the alternative to the $93-\mathrm{gm}$. interpolation was only 77 gm., viz., for the 77-93 condition. Insofar as the 77-93 Ss balance their judgment frequencies, their PSEs increase. As shown in Table 1, the PSE following the 77-gm. interpolation for the 77-93 condition was actually higher than the PSE following the 93-gm. interpolation for the 93-190 condition.

\section{A frequency model}

The tendency toward equal response frequency can be added to Helson's weighted-average model if a distinction is made between PSE and the remembered value of the standard. In accordance with the present interpretation of adaptation-level theory, this remembered value (M) would itself be a weighted average of the various stimuli affecting it, including the standard, comparison values, and past stimuli not manipulated in the present experiment. However, PSE would be shifted from $M$ toward the particular value $(F)$ that it would have had if the categories had been used with equal frequency. With no interpolations:

$$
P S E=a M+(1-a) F .
$$

In the present experiment, $\mathbf{F}=\mathbf{1 0 5}$ gm., the median value of the comparison stimuli.

With a single interpolated stimulus $\left(I_{S}\right)$, the remembered value of the standard $\left(M_{S}\right)$ is a weighted average of what it would have been without interpolation and the interpolated value:

$$
M_{S}=b M+(1-b) I_{S}
$$

so that substituting $\mathrm{M}_{\mathrm{S}}$ from Equation 3 for $\mathrm{M}$ in Equation 2:

$$
P S E_{s}=a\left[b M+(1-b) I_{s}\right]+(1-a) F .
$$

With two interpolated stimuli ( $I_{1}$ and $I_{2}$ ), the remembered value of the standard on trials using $I_{1}$ is given by:

$$
M_{1}=b M+(1-b)\left[c I_{1}+(1-c) I_{2}\right]
$$

and on the remaining trials by:

$$
M_{2}=b M+(1-b)\left[c I_{2}+(1-c) I_{1}\right] \text {. }
$$

The PSE on $I_{1}$ trials (PSE 1 ) is the remembered value of the standard on that kind of trial $\left(M_{1}\right)$ modified by the tendency to use the categories with equal frequency over the whole series of $I_{1}$ and $I_{2}$ trials. For the present experiment, in which equally-spaced comparison stimuli were presented with equal frequency, the overall category frequencies can be equal only when the mean of the two remembered values corresponds to the equal-frequency value, i.e., when $\left(M_{1}+M_{2}\right) / 2=F$. Insofar as the two remembered values are not centered around $F$, it is assumed that the frequency tendency shifts both $\mathrm{PSE}_{1}$ and $\mathrm{PSE}_{2}$ in the same direction and by an amount proportional to the difference between $F$ and $\left(M_{1}+M_{2}\right) / 2$. The constant of proportionality (1-a) represents the relative effect of the frequency tendency so that:

$$
\begin{aligned}
& \mathrm{PSE}_{1}=M_{1}+(1-a)\left[F-\left(M_{1}+M_{2}\right) / 2\right], \text { and } \\
& \mathrm{PSE}_{2}=M_{2}+(1-a)\left[F-\left(M_{1}+M_{2}\right) / 2\right) .
\end{aligned}
$$

If $\mathrm{a}=0$, the categories would be used with equal frequency since $\left(\mathrm{PSE}_{1}+\mathrm{PSE}_{2}\right) / 2=\mathrm{F}$; if $\mathrm{a}=1, \mathrm{PSE}_{1}=\mathrm{M}_{1}$ and Equations 7 and 8 reduce to the same form as Equation 1, the simple averaging model for adaptation level.

Substituting $\mathbf{M}_{1}$ and $\mathbf{M}_{2}$ from Equations $\mathbf{5}$ and 6 into 7:

$$
\begin{gathered}
P S E_{1}=a b M+(1-a) \mathrm{F}+(1-b)[c-(1-a) / 2] \mathrm{I}_{1}+ \\
(1-b)[(1-c)-(1-a) / 2] \mathrm{I}_{2}
\end{gathered}
$$

With only $I_{1}$ and $I_{2}$ varying in the present experiment, Equation 9 has the form of a standard regression equation:

$$
\mathrm{PSE}_{1}=\alpha+\beta_{1} \mathrm{I}_{1}+\beta_{2} \mathrm{I}_{2}
$$

The PSEs from all but the control condition were used in a multiple regression analysis, with $I_{1}=I_{2}$ for the two conditions with a single interpolated stimulus. The multiple correlation coefficient is .95 , no deviation exceeding two standard errors of the obtained PSE. Since the same regression equation fits the simple 
adaptation-level model as described by Equation 1, comparison of the models must be directed to the best-fit values of the empirical constants.

For the simple adaptation-level model, the constants $a$ and $b$ in Equation 1 are given directly by the solutions for the two $\beta$ coefficients: $\beta_{1}=a=.125, \beta_{2}=b=-.0585$ (with $\alpha=95.6$ ). This means that the best-fit weighting of one of the interpolated stimuli is negative (and significantly so, $\mathrm{p}<.01$ ). In terms of the simple model, the data thus appear inconsistent with the adaptationlevel principle that each stimulus, insofar as it has any effect, pulls adaptation level toward its own value.

For the frequency model as described by Equation 9 , the regression equation does not yield unique values of $a, b, c$, and $M$-only the relationships between them. However, the values of each of the three weighting constants are fixed for any value of $M$. Thus, if $M=$ $94 \mathrm{gm}$., then $\mathrm{a}=.29, \mathrm{~b}=.77, \mathrm{c}=.89$, and the remembered values of the standard are those given in the last column of Table 1. This example is just one of a theoretically infinite set, each of whose members would be equally consistent with the data. The importance of this example is that it demonstrates how the frequency model can yield remembered values for the standard thatare consistent with the assumption that each stimulus pulls memory toward its own value. Although the empirical PSEs sometimes vary inversely with the value of the interpolated stimuli, this is never true for the remembered values that are described by the frequency model. Thus, the differences between the remembered values for conditions with a single interpolation, 93-Only and 190-Only, and those for either the $93-\mathrm{gm}$. or $190-\mathrm{gm}$. trials in the 93-190 and 77-93 conditions are all in the direction required by a weighted-average model for memory.

Although the data from this experiment do not provide the most powerful test of the frequency model, they do suggest that it improves upon the simpler adaptation-level model. In particular, the direction of the effect of a second interpolated weight is inconsistent with the adaptation-level approach unless the proposed representation of the tendency toward balanced use of the responses is added. A more rigorous test of this added feature would require presenting comparison stimuli with varying frequencies so that the equalfrequency value (F) would vary over different conditions. This value can be calculated for any distribution of comparison frequencies by a method of successive approximation (described in Parducci, 1965). Using the calculated values of $F$ as a third predictor variable, a unique solution could be obtained for $a$, and hence for the other three parameters. In keeping with the averaging model for memory, it is crucial that the comparison stimuli have the same mean value for each value of F. Otherwise, there would also be shifts in $M$, which represents the pooled effects of the stimuli that were constant in the present experiment.

The proposed modification resembles the rangefrequency model used to describe the effects of stimulus context upon absolute category judgments (Parducci, 1965). Both models assume that the limen shifts toward the value it would have if the categories were used with equal frequency. However, the psychological range of stimulation has a major effect upon the location of the category limens when there is no standard, each category corresponding to a subrange that is independent of the stimulus frequencies. The present model assumes that the range, apart from its effect upon $M$ and $F$, is not important when there is a standard. Instead, the model uses the basic notion of adaptation-level theory: the net effects of different stimulus classes such as the standard, present and past interpolations, and the comparison stimuli can be presented as a weighted mean of the stimulus values. This determines the remembered value of the standard.

\section{CONCLUSIONS}

How is memory for a psychophysical stimulus affected by similar, interfering stimuli? The answer is not simple if one identifies memory with PSE, the comparison value judged equal to the previously presented standard. However, if PSE also reflects an equal-frequency tendency, as described by the present frequency model, then the answer is that memory shifts toward the value of each of the other stimuli. The magnitude of the shift differs for different classes of stimuli. But for each class, the shift is proportional to the difference between the interfering value and the value the memory would otherwise have had.

\section{References}

Femberger, S. W. Interdependence of judgments within the series for the method of constant stimuli. J. exp. Psychol., 1920, 3, $126-150$.

Guilford, J. P. Psychometric methods. New York: McGraw-Hill, 1954.

Harris, J. D. Discrimination of pitch: Suggestions toward method and procedure. Amer. J. Psychol., 1948, 61, 309-322.

Helson, H. Adaptation-level theory. New York: Harper \& Row, 1964.

Parducci, A. Range-frequency compromise in judgment. Psychol. Monogr., 1963, 77, No. 2, Whole No. 565.

Parducci, A. Category judgment: A range-frequency model. Psychol. Rev., 1965, 72, 407-418.

Swets, J. A., Tanner, W. P., \& Birdsall, T. G. Decision processes in perception. Psychol. Rev., 1961, 68, 301-340.

\section{Note}

1. This research was supported by Public Health Service Grant HD-00923 and National Science Foundation Grant GB-1768.

(Received in the Editorial Office December $30,1965$. 